\title{
Strategies to limit immune-activation in HIV patients
}

\section{Alessandra Bandera, Elisa Colella, Giuliano Rizzardini, Andrea Gori \& Mario Clerici}

To cite this article: Alessandra Bandera, Elisa Colella, Giuliano Rizzardini, Andrea Gori \& Mario Clerici (2016): Strategies to limit immune-activation in HIV patients, Expert Review of Antiinfective Therapy, DOI: $10.1080 / 14787210.2017 .1250624$

To link to this article: http://dx.doi.org/10.1080/14787210.2017.1250624

Accepted author version posted online: 20

Oct 2016.

Published online: 04 Nov 2016.

Submit your article to this journal $2 \pi$

Џ Article views: 27

View related articles \ulcorner

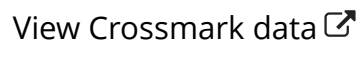




\title{
Strategies to limit immune-activation in HIV patients
}

\author{
Alessandra Bandera ${ }^{\mathrm{a}}$, Elisa Colella ${ }^{\mathrm{a}}$, Giuliano Rizzardini ${ }^{\mathrm{b}, \mathrm{c}}$, Andrea Gori $^{\mathrm{a}}$ and Mario Clerici ${ }^{\mathrm{d}, \mathrm{e}}$ \\ ${ }^{a}$ Clinic of Infectious Diseases, 'San Gerardo' Hospital - ASST Monza, School of Medicine and Surgery, University Milano-Bicocca, Monza, Italy; \\ ${ }^{b}$ Department of Infectious Diseases, ASST Fatebenefratelli Sacco, Milano, Italy; 'School of Clinical Medicine, Faculty of Health Science, University of

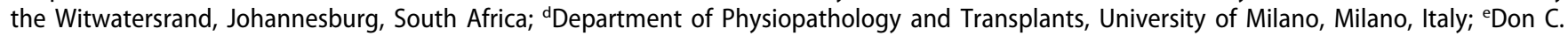 \\ Gnocchi Foundation, Istituto di Ricovero e Cura a Carattere Scientifico [IRCCS], Milano, Italy
}

\section{ABSTRACT}

Introduction: Antiretroviral treatment of HIV infection reduces, but does not eliminate, viral replication and down modulates immune activation. The persistence of low level HIV replication in the host, nevertheless, drives a smouldering degree of immune activation that is observed throughout the natural history of disease and is the main driving force sustaining morbidity and mortality.

Areas covered: Early start of antiretroviral therapy (ART) and intensive management of behavioural risk factors are possible but, at best, marginally successful ways to manage immune activation. We review alternative, possible strategies to reduce immune activation in HIV infection including timing of ART initiation and ART intensification to reduce HIV residual viremia; switch of ART to newer molecules with reduced toxicity; use of anti inflammatory/immunomodulatory agents and, finally, interventions aimed at modifying the composition of the microbiota.

Expert commentary: Current therapeutic strategies to limit immune activation are only marginally successful. Because HIV eradication is currently impossible, intensive studies are needed to determine if and how immune activation can be silenced in HIV infection.
ARTICLE HISTORY

Received 6 July 2016 Accepted 17 October 2016

KEYWORDS

HIV; immune activation; antiretroviral therapy; microbial translocation; comorbidity

\section{Introduction}

Antiretroviral therapy (ART) almost inevitably results in the rapid control of HIV and a partial restoration of immune responses, leading to the prevention of the various complications that define AIDS. However, HIV-infected adults experiencing durable treatment-mediated suppression of HIV replication are at risk for developing a number of non-AIDS conditions, including cardiovascular disease (CVD), cancer, kidney disease, liver disease, osteopenia/ osteoporosis, and neurocognitive disease (collectively referred to as 'serious non-AIDS events'). Although these events have a complex pathogenesis, low-grade chronic immune activation together with the direct effect of HIV, the impact of immunodeficiency, and ART toxicity - has convincingly been shown to be the main driver of non-AIDS pathologies. The complexity of the pathogenesis of non-AIDS events in HIV-infected individuals who have apparently achieved suppression of viral replication is exemplified by the observation that even the phenomenon defined as 'immune activation' is characterized by many different aspects. Thus, activation is only one manifestation of a complex immunological disorder that includes both immunosuppression and aspects of excessive inflammatory answers [1,2].

HIV infection leads to activation of both innate and adaptive immune responses through multiple mechanisms including (1) plasmacytoid dendritic cell stimulation by HIV-RNA; (2) stimulation of dendritic cells, natural killer (NK) cells, cytotoxic cell function, as well as antibody production and permanent CD8 T-cell dysfunction/exhaustion most likely due to the persistence of HIV antigens; (3) pyroptosis, an inflammatory form of programmed cell death resulting in the release of cytoplasmic contents and pro-inflammatory cytokines which is presumably triggered by abortive HIV infection of CD4+ T cells [3-5]; and (4) HIV persistence, a phenomenon mostly involving CD4+T lymphocytes that express the programmed cell death 1 (PDCD1; also known as PD1) receptor and are localized in the lymph nodes (notably, these cells are believed to be the principal source of replication-competent HIV-1 and of infectious virus [6]).

CD4+ T cells in gut-associated lymphoid tissue are known to be major targets for HIV due to their activated status and their high expression of $\mathrm{C}-\mathrm{C}$ chemokine receptor type 5 (CCR5) [7]. CD4+ T lymphocytes belonging to the Th17 subpopulation, in particular, are preferentially lost in initial HIV infection [8]. Because these cells secrete interleukin (IL)-17 and IL-22 and promote neutrophil recruitment, which is associated with resistance against bacterial and fungal infections and with the preservation of the integrity of the epithelial barrier, such loss plays a pivotal role in the pathogenesis of the disease. Recent results have shown that Th22 cells, another CD4+ T-cell subset that secretes IL-22 independently of interferon (IFN) gamma and IL-17, are selectively deleted as well in patients with uncontrolled HIV infection. Importantly, Th22 depletion is negatively correlated with immune activation and with microbial translocation [9]. At the gut level, HIV infection also leads to the disruption of intestinal tight junctions and increased mucosal permeability [10]; the consequent translocation of bacterial and fungal products elicits further inflammatory responses. An additional element that may play an important 
role in the HIV-associated subversion of the immune system is the alteration of the microbiota that is evident even in the initial phases of infection. Indeed, some studies in humans have shown that microbial richness is negatively and precociously altered by HIV infection. A shift from a Bacteroides- to a Prevotella-dominated scenario, in particular, was described to characterize HIV-1 infection starting from the initial phases of the disease $[11,12]$.

HIV infection-associated CD4+ T-cell depletion also triggers physiologic homeostatic mechanisms, e.g. IL-7 secretion [13] that result in lymphocyte proliferation. This stimulates the differentiation and the generation of effector T cells characterized by an inflammatory phenotype, thereby contributing to the persistence of immune activation [14]. Finally, and to add further complexity to this scenario, it also has to be underlined that other, non-immunologic or virologic risk factors, including smoke, alteration of lipid profile, and ART toxicity, play an important role in the pathogenesis of inflammation and immune activation in HIV-infected hosts $[15,16]$.

Herein, we will summarize the possible roles of the known culprits of immune activation in HIV infection, and we will focus on the state-of-the-art of the possible strategies to limit this deleterious condition.

\section{Strategies to limit immune activation in HIV patients}

\subsection{ART initiation}

The most important and simplest way to reduce immune activation is certainly ART. As a matter of fact, ART suppresses HIV viremia and consequently reduces immune activation. Nevertheless, even if ART results in control over viral replication, its effects over immune activation are only partial, as a low-grade and persistent degree of immune activation (i.e. increased amounts of circulating activated immune cells and an upregulated generation of inflammatory cytokines) is present throughout the duration of the disease [17]. Notably, ART itself cannot be deemed free from side effects, especially when considering older-generation protease inhibitors and thymidine analog nucleoside reverse-transcriptase inhibitors (NRTIs), drugs that are associated with lipodystrophy, insulin resistance, and dyslipidemia $[15,16]$.

Despite these considerations, early ART initiation has repeatedly and convincingly been shown to result in a significant reduction of serious non-AIDS events even in patients who start ART when their CD4+ T lymphocyte counts are next to normal. Early ART initiation, i.e. initiation of ART as soon as possible after the diagnosis of HIV infection, results in smaller HIV-DNA reservoirs and a lower degree of CD4+ T-cell activation, a parameter which was shown to be associated with preART CD4+ T-cell counts rather than with HIV viremia. Because most HIV-infected patients are first seen in the chronic phase of infection, early ART initiation is nevertheless often impossible in the real world; the best strategy in this case appears to be summarized by the 'test and treat' approach $[18,19]$.

A very important contribution to the concept that early initiation of ART is beneficial for patients was given by the results of the Strategic Timing of Antiretroviral Therapy
(START) protocol. This huge multicentric international trial enrolled more than 4000 naive patients that were followed for a mean of 3.0 years and showed that early ART initiation results in a lower incidence of both AIDS- and non-AIDSrelated events. Thus, the results of START clearly indicated that precocious initiation of therapy is associated with an important beneficial effect on disease outcome which is independent of age, sex, race, region of the world, CD4+ count, viral load, or risk factors for serious non-AIDS diseases. It is nevertheless important to observe that, even in the case of very early ART initiation, the risk of AIDS is not equal to zero, strongly indicating that irreversible immune system damages are present even in the very early stages of HIV infection [18].

A number of data have convincingly shown that higher levels of immune activation and lymphocytes apoptosis are present in treatment-naive patients with low CD4+ counts; these parameters decrease as a result of ART initiation [2022]. Notably, a recent study focusing on a small group of HIV controllers (both elite controller and patients with $<1000$ copies/milliliter $(\mathrm{cp} / \mathrm{mL})$ HIV-RNA for $>12$ months in the absence of ART) showed that lower HIV-RNA levels and HIV antibody titers, as well as a downmodulation of immune activation, can be achieved even in such hyperselected patients upon ART initiation [23]. Because the immune system of these particular individuals has repeatedly been shown to be only marginally damaged by HIV infection, these results support the idea that better preserved immune functions result in a more favorable response to ART.

To summarize, a vast body of literature shows that early initiation of ART is associated with a beneficial effect on disease outcome independently of the immuno-virological status of the patient. Even in this case, though, subtle and diffuse alterations that affect the immune system and cannot be fully restored by therapy are observed.

\subsection{ART intensification}

Many studies have shown that persistent HIV replication, even when ART suppresses HIV viremia below detection limit, is associated with a residual degree of immune activation. This is witnessed by the observations that higher amounts of CD4 +- and CD8+-activated T lymphocytes as well as higher plasma concentration of IL-6, D-dimer, and SCD14 are present even in those patients whose virological response to therapy can be classified as being optimal. As indicated above, these observations are of extreme clinical importance, as they result in an increased incidence of serious non-AIDS events [24].

One of the simplest and more logical way to curb immune activation in ART-treated individuals is to intensify therapy in the attempt to achieve an even more complete suppression of HIV replication. Different antiviral compounds that have been used in therapy intensification are mentioned below.

\subsubsection{Intensification with raltegravir}

The effect of intensification of ongoing ART with raltegravir, a potent integrase inhibitor, has been tested in a number of clinical trials. None of these trials, nevertheless, could convincingly show that this drug resulted in a significant reduction in plasma HIVRNA as measured by ultrasensitive methods. Studies dealing 
with inflammation and immune activation markers have shown conflicting results: although some studies noted a reduction in D-dimer levels and T-cell activation, as well as an early transient increase in 2-Long Terminal Repeat (2-LTR) circles (i.e. viral DNA that does not integrate into the host cell) post-raltegravir intensification, these effects could not be confirmed by other investigators [25-30].

\subsubsection{Intensification with maraviroc}

Maraviroc is an entry inhibitor that targets CCR5. Maraviroc intensification studies reached conflicting data as well: some studies found a reduction of CD4+ and CD8+ T lymphocytes bearing activation markers, while other studies showed no difference or even an increase of these cell populations [31-33]. Some interesting results stem from a pilot study showing that intensification with maraviroc results in the normalization of mucosal CCR5+CD4+ T cells, an increase of the naive/memory CD8+ T-cell ratio, and a decline of sCD14 levels and duodenal HIV DNA levels, with no changes in HIV-RNA in plasma or tissue. This particular study was conducted in naive patients treated with a quadruple regimen, containing an NRTI backbone (tenofovir disoproxil fumarate/emtricitabine) associated with maraviroc and raltegravir [34]. The optimism raised by these data was nevertheless at least partially dampened by other recent results indicating no differences in HIV-reservoir size in blood and sigmoid colon and in immune activation markers when a standard ART was compared with mega-ART (i.e. standard ART intensified with raltegravir/maraviroc) in acute infection [35].

To summarize, current data do not support the idea that therapy intensification of an effective and suppressive antiretroviral regimen does result in clear immunologic, virologic, or clinical benefits.

\subsection{HIV persistence and the way to eradication}

HIV low-level viremia is associated with microbial translocation and inflammation. The relationship between persistent viremia and inflammation is particularly intricate as inflammation contributes to HIV-1 persistence by inducing de novo infection in activated $\mathrm{CD}^{+} \mathrm{T}$ cells and by upregulating the expression of immune checkpoint blockers and of immune proteins (e.g. PD1) that blunt HIV-1-specific immune responses. Persistent viral replication, in turn, is a major factor in the maintenance of a pro-inflammatory microenvironment [6,36-53].

Recent results casted a new light on the problem of HIV persistence. PD-1 expressing CD4+ $T$ cells in the lymph nodes (LN PD-1 $1^{+} / \mathrm{T}_{\mathrm{FH}}$ cells), in particular, were shown to harbor cell-associated HIV-RNA for up to 12 years after initiation of ART, possibly because of their location in the germinal centers, which are a privileged site for virus replication and infection [43]. These results suggested that therapy based on the use of PD-1-specific antibodies might facilitate the elimination of these cells, greatly reducing the pool of latent HIV-1 and, as a consequence, 'curing' immune activation $[6,44,45]$. An additional, extremely important factor that obstacles the possibility of achieving viral eradication is the existence of so-called 'sanctuaries: anatomic compartments where drug concentrations are lower than in blood.' Some examples of such compartments are the brain, the testes, the lungs, and the lymphoid tissue. In sanctuaries, HIV can replicate and evolve while being undetectable in the bloodstream for long periods of time $[46,47]$. At the moment, this problem remains unsolved and in dire need for targeted research.

\subsection{Switch of ART regimens}

With the development of new and more user-friendly antiretroviral drugs, ART-associated toxicity has become less frequent, as this problem can be bypassed by skipping those drugs whose use is more frequently burdened by toxicity. Thus, the availability of many antiviral compounds allows the clinician to avoid using older drugs that are known to be associated with metabolic dysfunction. In particular, old-generation NRTIs, such as zidovudine and stavudine, as well as older protease inhibitors, are known to be associated with lipodystrophy and dyslipidemia, and their use is currently avoided unless it becomes strictly necessary [48].

As a consequence of such realization, a useful strategy is to switch from ART to newer molecules: several studies demonstrate an improvement in lipid profile once patients switch from old protease inhibitors to darunavir or atazanavir. Therapy simplification, e.g. a switch from standard triple therapy to dual-therapy containing a protease inhibitor such as lopinavir or atazanavir, associated with lamivudine $[49,50]$ can reduce toxicity problems as well. Other useful solutions are the use of antiretroviral regimens that include integrase inhibitors or CCR5 antagonists, i.e. compounds that are characterized by more favorable lipid profiles $[51,52]$.

In this context, it should be noted that tenofovir disoproxil fumarate-based regimens are also associated with a more favorable lipid profile, but the observations that this drug results in an increased risk of reduced bone mineral density and estimated Glomerular Filtration Rate requires a careful evaluation of risks and benefits for each patient [48]. These considerations are extremely important within the immune activation scenario. Thus, the use of older drugs was often associated with mitochondrial damage, dyslipidemia, and metabolic disorders, all factors that play an important role in oxidative stress and inflammation: switch to newer, 'cleaner' drugs is an effective and beneficial way to reduce immune activation.

\subsection{Anti-inflammatory agents}

An obvious way to downmodulate inflammation and immune activation is to use anti-inflammatory agents. Several molecules endowed with different degrees of anti-inflammatory effects have been studied in the context of HIV infection.

\subsubsection{Statins}

From a classical point of view, statins have a lipid-lowering effect which is extremely useful for primary or secondary cardiovascular prevention [53]. However, many studies showed a pleiotropic effect of these drugs, which deserves a careful analysis. Statins play an important role against oxidative stress, endothelial dysfunction, and vascular inflammation: potential 
therapeutic targets when the renin-angiotensin-aldosterone system is activated by accumulation of conventional cardiovascular risk factors [54].

Even more interesting, statins have an immunomodulatory effect which is mediated by different mechanisms: (1) inhibition of IFN- $\gamma$ co-stimulation-dependent expression of human leukocyte antigen (HLA) class II antigens on macrophages; (2) inhibition of the expression of co-stimulatory molecules (CD40, CD8, and CD86) on antigen-presenting cells; (3) reduction of Th1 cytokine production; and (4) stimulation of the secretion of Th2 cytokines [55].

In an interesting recent work, atorvastatin was shown to be endowed with many anti-inflammatory functions including the reduction of T-cell immune activation markers (e.g. CD38, HLA-DR, and Ki67) and of the expression of the HIV-1 Coreceptor CCR5, as well as the decrease of the proliferative capabilities of CD4+ T cells in vitro. Moreover, atorvastatin resulted in (1) the expansion of regulatory T cells (Treg); (2) the expression on CD4+ T lymphocytes of T-cell immunoglobulin and immunoreceptor tyrosine-based inhibition motif domain - a molecule that stimulates the suppressive activity of Tregs -; and (3) the upregulation, on these same cells, of the cyclin-dependent kinase inhibitor p21, a protein that renders them less susceptible to HIV-1 infection [56].

Clinical trials analyzing the effects of statins on immune activation in HIV infection showed that these compounds can reduce serum levels of C-reactive protein (CRP) with or without an effect on other immune activation markers. Some of these discrepancies could be related to the different statins used in these studies, as well as to differences among the analyzed populations of HIV patients $[57,58]$.

The possibility of employing statins as anti-inflammatory agents in HIV-infected individuals is, thus, a hot research topic. Two interesting atorvastatin-based, randomized, double-blind, placebo-controlled trials showed a reduction of activated CD4 + and CD8+ T lymphocytes in ART-treated individuals $[59,60]$. Even more recently, the use of atorvastatin and rosuvastatin in HIV infection was shown to reduce oxidized low-density lipoprotein (oxLDL) levels, carotid intima media thickness, coronary atherosclerosis, and monocyte activation [61,62].

The ability of another statin, rosuvastatin, to improve cardiovascular and skeletal health in HIV infection by simultaneously targeting inflammation and dyslipidemia is currently being evaluated in the Stopping Atherosclerosis and Treating Unhealthy bone with RosuvastatiN in HIV trial. Preliminary data indicate that rosuvastatin can reduce monocyte activation and the concentration of sCD14, as well as CD142 expression on monocytes, independently of its lipid-lowering effects [63]. This activity is also associated with increased bone mineral density [64]. Importantly, rosuvastatin also reduced intestinal fatty acid-binding protein, a marker of enterocyte death and a surrogate marker of gut-barrier integrity, even if its use did not affect serum levels of lipopolysaccharide (LPS)binding protein (LBP), a marker of microbial translocation [65].

\subsubsection{Aspirin}

Acetylsalicylic acid has a fundamental role in secondary prevention of CVD [66]. The results of a pilot study proved that a short-course acetylsalicylic acid therapy in a small group of
HIV-positive patient on ART was associated with reductions in platelet aggregation, CD4+ and CD8+ T-cell activation, and plasma sCD14 levels [67]. A second interesting study focused on the possible effects of aspirin on nuclear factor kappa-lightchain-enhancer of activated B cells, a transcription factor that plays an important role in inflammation and is constitutively activated in several types of cancers, including Epstein-Barr Virus-positive lymphoma. Results indicated that aspirin reduced nuclear translocation of $\mathrm{NFKB}$ and promoted the lytic cycle. These data suggest that acetylsalicylic acid could be used, of course in combination with anticancer drugs, in the treatment of EBV-positive lymphomas [68]. Notably, as NFKB is constitutively activated in Kaposi's sarcoma-associated herpes virus (KSHV) and primary effusion lymphoma, NFK $\beta$ inhibitors could also play a role in the therapy of these conditions [69]. This is confirmed by two small studies showing that aspirin-mediated NFK $\beta$ inhibition provokes the apoptosis of KSHV-infected cells, possibly resulting in a beneficial clinical effect $[69,70]$.

\subsubsection{Hydroxychloroquine}

Chloroquine (CQ) and its analog hydroxychloroquine (HCQ) have shown both immunomodulatory and anti-HIV properties.

Several mechanisms of action of these antimalarial compounds on the immune system have been proposed: (1) interference with lysosomal acidification and inhibition of proteolysis, chemotaxis, phagocytosis, and antigen presentation [71]; (2) reduction of macrophage-mediated cytokine production (in particular IL-1 and IL-6 production) [72]; (3) inhibition of phospholipase A2 with a consequent antagonizing effect on prostaglandins [73]; (4) absorption and block of ultraviole light-induced cutaneous reactions; (5) binding and stabilization of DNA [74]; (6) inhibition of T- and B-cell receptor-mediated calcium signaling; (7) inhibition of matrix metalloproteinases [75]; and (8) inhibition of toll-like receptor signaling [76].

Mechanisms that have been invoked to explain the antiHIV-1 effect of CQ and HCQ include an impairment of gp120 production, the restriction of intracellular iron which is a necessary cofactor for HIV-1 replication, an effect on Tatmediated transactivation of HIV-1 LTR, and, finally, an effect on HIV-1 integrase [77,78]. Two non-randomized studies analyzing the possible effects of $\mathrm{HCQ}$ on immune activation in HIV-positive patients showed conflicting results. Thus, the first study suggested that the use of HCQ is associated with a sharp reduction in plasma LPS, IL-6, and activated T cells and monocytes, while the second study found no differences in lymphoid and myeloid immune activation or inflammatory biomarkers $[79,80]$. These two studies nevertheless cannot be compared as the first one was conducted using a higher dose of HCQ in virologically non-suppressed individuals, whereas the second focused on HIV-suppressed patients.

\subsubsection{Agents preventing monocyte activation}

HIV-infected monocytes and macrophages present in anatomic reservoirs, including tissues such as the brain and lung, can escape immune system recognition, thus establishing viral reservoirs [81]. Monocytes play a role in many clinical manifestations, such as neuro-AIDS. Notably, persistently 
increased numbers and/or percentages of CD16+ monocytes are more tightly linked to the development of neurological diseases than the number of HIV-infected cells in the Central Nervous System or Cerebrospinal Fluid viral load [82].

Monocyte expression of CD11b and CX3CR1 was recently shown to associate with carotid intima-media thickness in HIV-infected individuals. Other studies indicate that the monocyte activation phenotype in HIV-infected patients is similar to that seen in uninfected individuals suffering from CVDs $[83,84]$. These results notwithstanding, the proportion of inflammatory CD16+ monocytes is increased in untreated HIV infection and predicts progression of coronary artery calcium, independent of traditional risk factors [85]. The use of specific antiretroviral drugs that efficiently target monocytes could thus be beneficial as it might prevent some comorbidities. The CCR5 inhibitor maraviroc can avoid infection of monocyte-derived macrophages ex vivo and, as a consequence, likely reduces the size of the reservoirs established in these cells in vivo. However, specific studies in HIV-infected individuals are currently lacking [86].

The integrase inhibitor raltegravir can reach therapeutic concentrations in the CSF and is equally potent in monocyte/macrophages and in lymphocytes [87]. This drug thus can target HIV-infected cells, including macrophages, in the central nervous system. It is, however, highly worrisome that, whereas multiple mutations are required to confer raltegravir resistance in T cells, a single mutation can achieve this deleterious result in macrophages [88]. A final word of caution stems from the observation that most of these data derive from studies conducted in HIV-uninfected individuals.

\subsection{Treating microbial translocation}

The use of ART does not result in the normalization of microbial translocation markers, including LPS and bacterial $16 \mathrm{~s}$ rDNA [89]. Microbial translocation is associated with the stimulation of mucosal innate and adaptive immune cells and therefore is a major driver of immune activation. LPS can also induce the expression of CD142 on monocytes [90]. Because CD142 triggers the coagulation cascade, and its expression on monocytes is correlated with D-dimer levels, persistent microbial translocation contributes to the coagulopathy and the increased incidence of CVD which are observed even in ARTtreated HIV-infected individuals [90].

During HIV infection, the balance of commensal bacterial communities is impaired, resulting in microbial dysbiosis, with alterations to the phyla Bacteroidetes, Firmicutes, and Proteobacteria, and the loss of beneficial bacterial genera, such as Bacteroides, Lactobacillus, and Bifidobacterium. Furthermore, the levels of several pathogenic Proteobacteria including those within the Campylobacter, Escherichia, Acinetobacter, Desulfovibrio, and Pseudomonas genera are increased during HIV infection [17,18,91-95]. Recent results showed that dysbiosis also correlates with activity of the kynurenine pathway of tryptophan catabolism, and tryptophan-degrading bacteria play a role in dysfunction of gut mucosal CD4 Th17/Th22 cells. This is likely a consequence of the immunosuppressive properties of kynurenine, a tryptophan metabolite, through indoleamine-2, 3-dioxygenase activity. Notably, the plasma kynurenine/tryptophan ratio is an independent predictor of mortality in HIV-infected patients initiating ART and may play a key role in HIV pathogenesis [96-101].

The observation that HIV infection results in a profound alteration of the microbiota suggests that the restoration of a physiological microbiota could results in beneficial effects on immune activation. Different strategies to treat dysbiosis have been analyzed.

\subsubsection{Prebiotics}

Prebiotics are compounds whose use can change the growth and/or activity of certain gut microflora, resulting in health benefits [102]. Prebiotics can modify host-microbe interactions via the microbiota and its metabolism, host epithelial, and other cells, as well as by influencing receptor expression and bacterial adhesion. Prebiotic oligosaccharides can also inhibit the adherence of specific pathogens to epithelial cells in vitro [103]. As indicated above, prebiotics are candidate agents to improve the intestinal homeostasis in HIV-infected individuals. Prebiotics do not contain bacteria but provide substrate for the intestinal microbiota [104]. Prebiotics can also reduce gastrointestinal infections, pathologies that are more prevalent in HIV-infected individuals [105]. Oligosaccharides are contained into bovine colostrum with other components, such as growth factors, immunoglobulins, and antimicrobial peptides, and have shown some activity in alleviating HIV-associated diarrhea in single-arm studies [106].

Results of a pilot study in ART-naive HIV-infected individuals showed that dietary supplementation with a prebiotic oligosaccharide mixture positively modified the composition of the microbiome, resulting in a reduction of SCD14 and of activated CD4+ T cells, as well as in improved NK cell activity [107]. A further study showed how a more prolonged use of these prebiotics was associated with a significant reduction of CD4+ T cell decline in HIV-infected ART-naive individuals [108]. An elder randomized controlled trial in which bovine colostrum was added to ART nevertheless found no differences in terms of CD4 T-cell count, microbial translocation markers, and T-cell activation markers [109]. As often is the case, these two results cannot be compared: the compounds used were different (colostrum vs. oligosaccharides) as were the groups of patients analyzed (ART suppressed vs. ART naive).

\subsubsection{Probiotics}

Probiotics are live microorganisms which, when administered in adequate amounts, confer a health benefit on the host [110]. Probiotics can interfere with the function and proliferation of pathogens in the gastrointestinal tract. Thus, probiotics can enhance the secretion of pathogen-specific IgA [111], induce $\beta$-defensin secretion [112], secrete bactericidal proteins [113], and reduce the adhesion and invasion of pathogens [114]. Antibiotic-like compounds, such as reuterin produced by Lactobacillus reuteri, exhibit broad-spectrum effects against Gram-positive and Gram-negative bacteria as well as fungi, yeast, and protozoa [113]. These characteristics could be beneficial in AIDS patients as $L$. reuteri was shown to prevent cryptosporidiosis in a murine AIDS model [115]. Moreover, it 
has been documented in different studies that a regular consumption of probiotics over a prolonged period could result in an improvement of CD4 T-cell count in HIV-positive patients [116-118]. Results of a recent clinical trial confirmed that the use of probiotics is associated with a significant decrease in activated CD4+ $T$ lymphocytes and a reduction of serum concentrations of high-sensitivity CRP, IL-6, and LBP [119]. A more complex study has been designed with the principal aim to analyze the effects of probiotics on immune activation, microbial translocation, composition of the microbiome, and safety, adherence, and tolerability in different HIV-infected patient groups. Results will likely be of great interest to the HIV community [120].

\subsubsection{Fecal transplantation}

A recent study has been conducted in Simian Immunodeficiency Virus-chronically infected and ART-treated rhesus macaques to analyze the safety, efficacy, and tolerability of fecal microbiota transplantation (FMT). Results showed that FMT resulted in an increased numbers of Th17 and Th22 cells as well as in a decreased activation of $\mathrm{CD}^{+} \mathrm{T}$ cells. Interestingly, these changes correlated most strongly across all sampling time points with a reduced abundance of taxonomic groups in the colon. The bacterial community composition at 2 weeks post-FMT resembled the pre-FMT community structure although differences in the abundances of minor bacterial populations remained [121].

These data suggest that FMT may have beneficial effects that should be further evaluated in larger studies, and they provide evidence that changes in the microbiome, particularly in terms of diversity and changes in minor populations, result in immune modulation and do not have adverse consequences [121].

\subsubsection{Sevelamer}

Sevelamer carbonate, a phosphate-lowering drug, decreases circulating LPS levels in patients with renal insufficiency, possibly by binding chylomicron-LPS complexes and preventing their reabsorption. In this population, sevelamer also reduces levels of sCD14, IL-6, CRP, and total and low-density lipoprotein cholesterol [122,123].

A small study on sevelamer in ART-naive HIV-infected people did not show decreases in microbial translocation, inflammation, or immune activation; however, its use was found to be associated with lower serum concentrations of tissue factor and oxLDL cholesterol, which may have beneficial cardiovascular effects [124]. Similar results were observed in SIVinfected nonhuman primates, in whom sevelamer reduced coagulation biomarkers [125].

This particular field of research is novel, promising, and interesting; larger and well-designed clinical studies are nevertheless needed to clarify if modification of the microbiome could have a therapeutic role in the treatment of HIV infection (Table 1).

\section{Conclusions}

Immune activation in HIV patients is an extremely complex issue; this phenomenon is likely responsible for HIV-associated and HIV-nonassociated complications of infection. Despite the efforts of the scientific community, the pathogenesis of immune activation is still not fully understood and, as a consequence, effective therapeutic strategies to prevent it/silence it are still not available. While some issues have been deeply studied, other aspects of immune activation require more analyses because of the lack of adequately powered trials or due to being conflicting results.

Currently available data focus attention on treatment of coinfection, such as hepatitis viruses, tuberculosis, as well as on management of traditional risk factor, including smoking, diabetes, hypertension, and hyperlipidemia. More studies are nevertheless required to clarify the potential benefits of other interventions, including the most promising ones: those targeting microbial translocation and reducing dysbiosis.

Over the years, we have reached two solid conclusions: (1) early initiation of therapy plays a fundamental role in reducing immune activation and (2) immune activation in HIV-infected individuals can be reduced but not abolished. As persistent low-degree immune activation (1) is present throughout the whole disease even in individuals in whom HIV replication is successfully suppressed; (2) is the main culprit of the non-AIDS events observed in HIV patients; (3) is most likely associated with low-grade viral replication; and (4) HIV eradication is currently impossible, major efforts will need to focus on better understanding the immunopathology of HIV disease with the final goal of curing it.

\section{Expert commentary}

HIV infection can be treated but not cured. ART suppresses viral replication but does not eradicate the virus. Ongoing lowrate HIV replication is the main culprit of the persistent immune activation seen even in successfully treated patients, and, on the other hand, immune activation plays a pivotal role in the pathogenesis of the non-AIDS events observed in HIV patients. Therapeutic strategies envisioned to 'cure' immune activation can be divided up into three groups: (1) antiviralbased (reduction of HIV load by earlier initiation of therapy and/or therapy intensification); (2) non-antiviral-based (immunomodulants and modifications of the microbiota); and (3) behavioral (reduction of risk factors, e.g. smoke and lipid profiles); none of these approaches has nevertheless reached univocal results. As HIV eradication, the only solution to this problem, is currently unachievable, intensive and smartly designed research is urgently needed to determine if and how immune activation can be silenced in HIV-infected individuals.

\section{Five-year view}

We believe that in the next 5 years, results of clinical studies will definitively demonstrate the clinical, immunological, and epidemiological advantages of early ART initiation. However, as the median age of people living with HIV increases, we will face a larger number of comorbidities and pathologies linked to aging. We will need to develop algorithms to manage the traditional risk factors for cardiovascular disease and tumors in an intensive and tailored way. We will also need to develop strategies to face new challenges, including those related with 


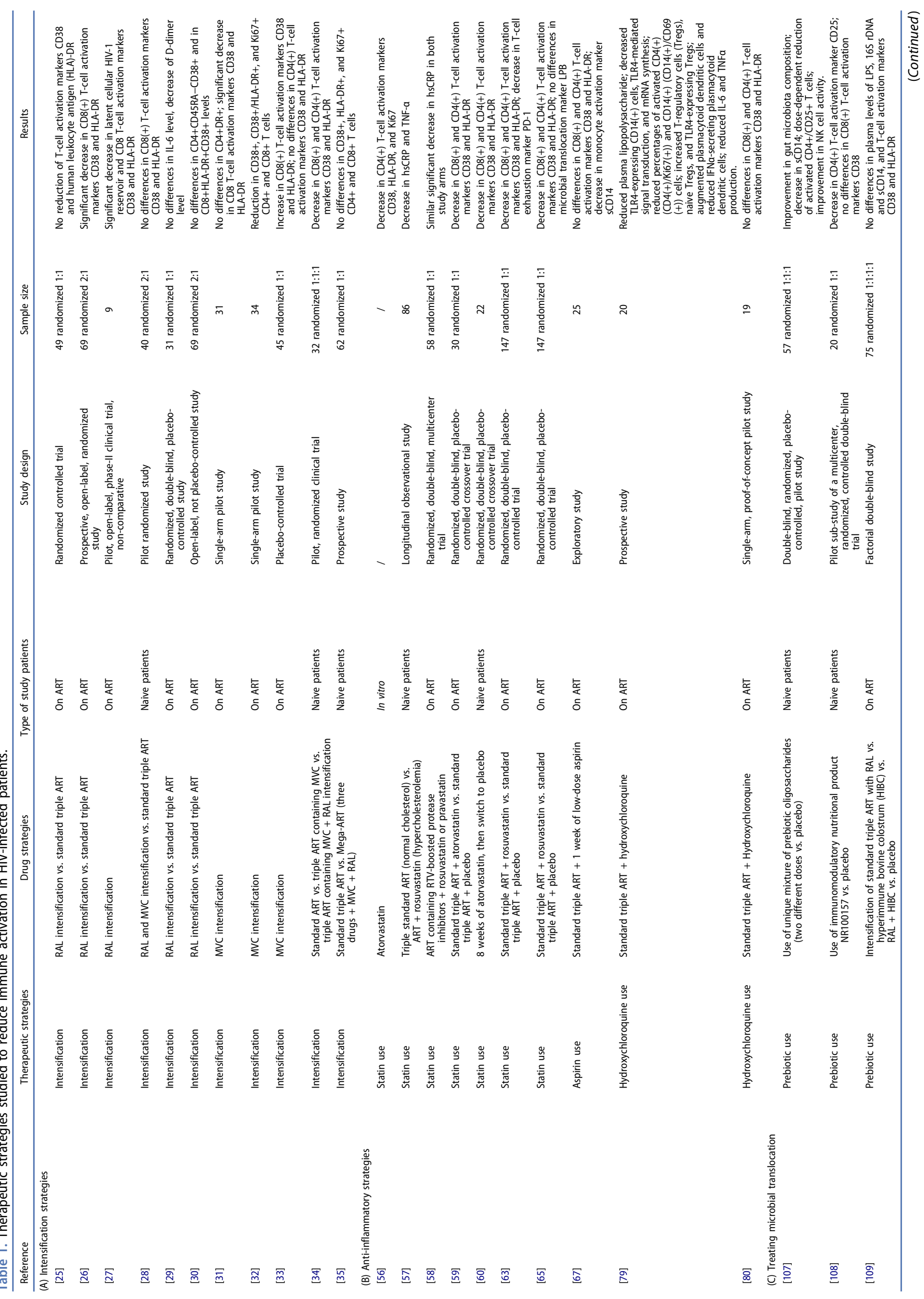




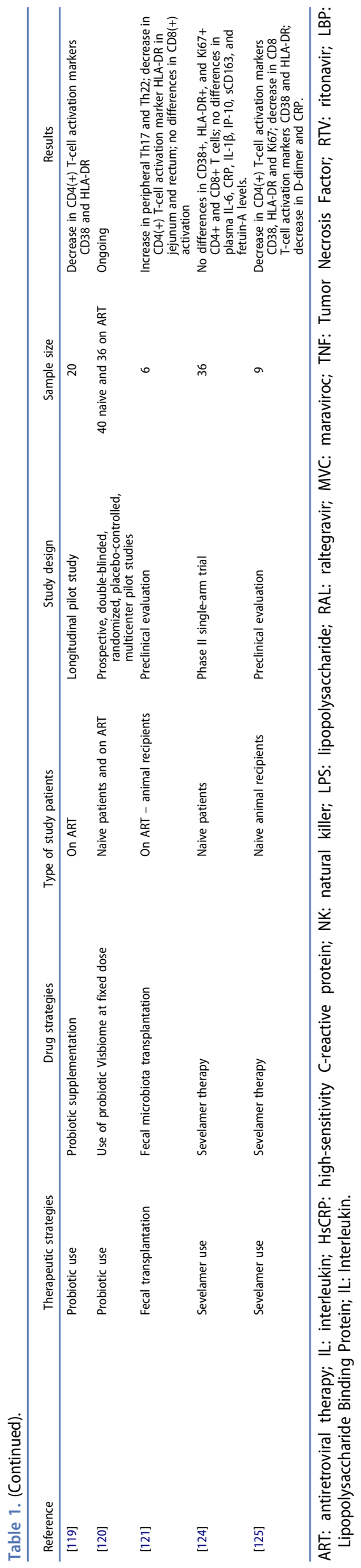

the higher incidence of neoplasia and of dementia as well as those stemming from frailty.

In the next 5 years, we will develop more appropriate diagnostic tools to measure immune activation and inflammation in ART-treated HIV-infected patients, as new strategies of nuclear medicine and/or magnetic resonance imaging will be introduced allowing us to better define the seize and the activity of viral reservoir. This new knowledge will also allow us to design clinical trials with the specific aim of hitting and, possibly, deleting such reservoirs. Finally, hopefully, we might develop efficacious immunomodulants that could allow us to specifically target HIV-associated immune alterations.

\section{Key issues}

- Thanks to modern antivirals HIV infection can be treated but cannot be cured. Succesful antiretroviral therapy suppresses HIV replication to undetecteble limits, but does not shut it down completely.

- Sneaky, smouldering viral replication persists and drives the low grade immune activation that accompaines the disease. This, in turn, is the major driver of the non-AIDS events observed in HIV patients.

- HIV eradication would take care of the problem, but is currently unachievable. Suppression of immune activation has been attempted through therapy intensification and interventins with different types of immune modulators (e.g. cloroquine, prebiotics, etc). None of these approaches has reached success.

- The fact that immune activation persists undettered in HIVinfected individuals witnesses the fact that our knowledge of the immunopathogenesis of this disease is still very partial and unsatisfactory.

- The old concept that antivirals take care of the virus but do not cure the patient is alive and well. Smart and intensive basic research on the immunology of HIV infection is nowadays only marginally financed, this is the negative consequence of the idea that the availability of antivirals coincides with the end of AIDS.

\section{Funding}

This paper was not funded.

\section{Declaration of interest}

The authors have no relevant affiliations or financial involvement with any organization or entity with a financial interest in or financial conflict with the subject matter or materials discussed in the manuscript. This includes employment, consultancies, honoraria, stock ownership or options, expert testimony, grants or patents received or pending, or royalties.

\section{References}

Papers of special note have been highlighted as either of interest $(\cdot)$ or of considerable interest (••) to readers.

1. Deeks SG, Lewin SR, Havlir DV. The end of AIDS: HIV infection as a chronic disease. Lancet. 2013 Nov 2;382(9903):1525-1533.

2. Palella FJ Jr, Baker RK, Moorman AC, et al. Mortality in the highly active antiretroviral therapy era: changing causes of death and 
disease in the HIV outpatient study. J Acquir Immune Defic Syndr. 2006;43(1):27-34.

3. Iwasaki A. Innate immune recognition of HIV-1. Immunity. 2012;37 (3):389-398.

4. Doitsh G, Galloway NL, Geng X, et al. Cell death by pyroptosis drives CD4 T-cell depletion in HIV-1 infection. Nature. 2014;505 (7484):509-514.

5. Hoffmann M, Pantazis N, Martin GE, et al. Exhaustion of activated CD8 T cells predicts disease progression in primary HIV-1 infection. PLoS Pathog. 2016 Jul 14;12(7):e1005661.

6. Banga R, Procopio FA, Noto A, et al. PD-1(+) and follicular helper T cells are responsible for persistent HIV-1 transcription in treated aviremic individuals. Nat Med. 2016 Jul;22(7):754-761.

7. Brenchley JM, Schacker TW, Ruff LE, et al. CD4+ T cell depletion during all stages of HIV disease occurs predominantly in the gastrointestinal tract. J Exp Med. 2004;200(6):749-759.

8. Brenchley JM, Paiardini M, Knox KS, et al. Differential Th17 CD4 T-cell depletion in pathogenic and nonpathogenic lentiviral infections. Blood. 2008;112(7):2826-2835.

9. Page EE, Greathead L, Metcalf R, et al. Loss of Th22 cells is associated with increased immune activation and IDO-1 activity in HIV-1 infection. J Acquir Immune Defic Syndr. 2014 Nov 1;67(3):227-235.

10. Nazli A, Chan O, Dobson-Belaire WN, et al. Exposure to HIV-1 directly impairs mucosal epithelial barrier integrity allowing microbial translocation. PLoS Pathog. 2010;6(4):e1000852.

11. Gori A, Tincati C, Rizzardini G, et al. Early impairment of gut function and gut flora supporting a role for alteration of gastrointestinal mucosa in human immunodeficiency virus pathogenesis. J Clin Microbiol. 2008;46(2):757-758.

12. Mutlu EA, Keshavarzian A, Losurdo J, et al. A compositional look at the human gastrointestinal microbiome and immune activation parameters in HIV infected subjects. PLoS Pathog. 2014;10(2): e1003829.

13. McCune JM, Napolitano LA, Grant RM, et al. Increased production of IL-7 accompanies HIV-1- mediated T-cell depletion: implications for T-cell homeostasis. Nat Med. 2001;7(1):73-79.

14. Catalfamo M, Di Mascio M, Hu Z, et al. HIV infection-associated immune activation occurs by two distinct pathways that differentially affect CD4 and CD8 T cells. Proc Natl Acad Sci USA. 2008;105 (50):19851-19856.

15. Carr A, Samaras K, Burton S, et al. A syndrome of peripheral lipodystrophy, hyperlipidaemia and insulin resistance in patients receiving HIV protease inhibitors. AIDS. 1998;12(7):F51-F58.

16. Mynarcik DC, McNurlan MA, Steigbigel RT, et al. Association of severe insulin resistance with both loss of limb fat and elevated serum tumor necrosis factor receptor levels in HIV lipodystrophy. J Acquir Immune Defic Syndr. 2000;25(4):312-321.

17 Wada NI, Jacobson LP, Margolick JB, et al. The effect of HAARTinduced HIV suppression on circulating markers of inflammation and immune activation. AIDS. 2015;29(4):463.

18. Group ISS Initiation of antiretroviral therapy in early asymptomatic HIV infection. N Engl J Med. 2015 Aug 27; 373(9): 795-807

-. This article is one of the main reference point for the antiretroviral national and international guidelines, and it holds many answers to many infectivological questions regarding HIV infection management.

19. Srinivasula S, Lempicki RA, Adelsberger JW, et al. Differential effects of HIV viral load and CD4 count on proliferation of naive and memory CD4 and CD8 T lymphocytes. Blood. 2011;118:262-270.

20. Soria A, Trabattoni D, Squillace N, et al. Prospective immune dynamics during the first 24 weeks of efavirenz based-antiretroviral therapy in HIV-1-infected subjects, according to CD4+ T-cell counts at presentation: the IMMUNEF clinical trial. PLoS One. 2015 Feb 11;10(2):e0117118.

21. Piconi S, Trabattoni D, Gori A, et al. Immune activation, apoptosis, and Treg activity are associated with persistently reduced CD4+ T-cell counts during antiretroviral therapy. AIDS. 2010;24:1991-2000.

22. Roger PM, Breittmayer JP, Durant J, et al. Early CD4(+) T cell recovery in human immunodeficiency virus-infected patients receiving effective therapy is related to a downregulation of apoptosis and not to proliferation. J Infect Dis. 2002;185:463-470.

23. Hatano $H$, Yukl SA, Ferre AL, et al. Prospective antiretroviral treatment of asymptomatic, HIV-1 infected controllers. PLoS Pathog. 2013;9(10):e1003691.

24. Armah KA, McGinnis K, Baker J, et al. HIV status, burden of comorbid disease, and biomarkers of inflammation, altered coagulation, and monocyte activation. Clin Infect Dis Off Publ Infect Dis Soc Am. 2012;55(1):126-136.

25. Gandhi RT, Zheng L, Bosch RJ, et al. The effect of raltegravir intensification on low-level residual viremia in HIV-infected patients on antiretroviral therapy: a randomized controlled trial. PLoS Med. 2010;7:8.

26. Llibre JM, Buzon MJ, Massanella M, et al. Treatment intensification with raltegravir in subjects with sustained HIV-1 viraemia suppression: a randomized 48-week study. Antivir Ther. 2012;17(2):355-364.

27. Vallejo A, Gutierrez C, Hernandez-Novoa B, et al. The effect of intensification with raltegravir on the HIV-1 reservoir of latently infected memory CD4 $T$ cells in suppressed patients. AIDS. 2012;26(15):1885-1894.

28. Markowitz M, Evering TH, Garmon D, et al. A randomized open-label study of 3- versus 5-drug combination antiretroviral therapy in newly HIV-1-infected individuals. J Acquir Immune Defic Syndr. 2014;66 (2):140-147.

29. Hatano H, Strain MC, Scherzer R, et al. Increase in 2-LTR circles and decrease in D-dimer after raltegravir intensification in treated HIVinfected patients: a randomized, placebo-controlled trial. J Infect Dis. 2013;208:1436-1442.

30. Buzón MJ, Massanella M, Llibre JM, et al. HIV-1 replication and immune dynamics are affected by raltegravir intensification of HAART-suppressed subjects. Nat Med. 2010;16(4):460-465.

31. Cuzin L, Trabelsi $S$, Delobel $P$, et al. Maraviroc intensification of stable antiviral therapy in HIV-1- infected patients with poor immune restoration: MARIMUNO-ANRS 145 study. J Acquir Immune Defic Syndr. 2012;61(5):557-564.

32. Wilkin TJ, Lalama CM, McKinnon J, et al. A pilot trial of adding maraviroc to suppressive antiretroviral therapy for suboptimal CD4 (+) T-cell recovery despite sustained virologic suppression: ACTG A5256. J Infect Dis. 2012;206(4):534-542.

33. Hunt PW, Shulman NS, Hayes TL, et al. The immunologic effects of maraviroc intensification in treated HIV-infected individuals with incomplete CD4+ T-cell recovery: a randomized trial. Blood. 2013;121(23):4635-4646.

34. Serrano-Villar S, Sainz T, Ma ZM, et al. Effects of combined CCR5/ integrase inhibitors-based regimen on mucosal immunity in HIVinfected patients naïve to antiretroviral therapy: a pilot randomized trial. PLoS Pathog. 2016 Jan 21;12(1):e1005381.

35. Ananworanich J, Chomont N, Fletcher JL, et al. Markers of HIV reservoir size and immune activation after treatment in acute HIV infection with and without raltegravir and maraviroc intensification. J Virus Erad. 2015;1(2):116-122.

36. Baroncelli S, Galluzzo CM, Pirillo MF, et al. Microbial translocation is associated with residual viral replication in HAART-treated HIV+ subjects with < 50copies/ml HIV-1 RNA. J Clin Virol. 2009 Dec;46 (4):367-370.

37. Hatano $H$, Jain V, Hunt PW, et al. Cell-based measures of viral persistence are associated with immune activation and programmed cell death protein 1 (PD-1)-expressing CD4+ T cells. J Infect Dis. 2013;208:50-56.

38. Klatt NR, Chomont N, Douek DC, et al. Immune activation and HIV persistence: implications for curative approaches to HIV infection. Immunol Rev. 2013;254:326-342.

39. Vandergeeten C, Fromentin R, DaFonseca S, et al. Interleukin-7 promotes HIV persistence during antiretroviral therapy. Blood. 2013;121:4321-4329.

40. Barouch DH, Deeks SG. Immunologic strategies for HIV-1 remission and eradication. Science. 2014;345:169-174.

41. Massanella M, Fromentin R, Chomont N. Residual inflammation and viral reservoirs: alliance against an HIV cure. Curr Opin HIV AIDS. 2016 Mar;11(2):234-241. 
42. Martinez-Picado J, Deeks SG. Persistent HIV-1 replication during antiretroviral therapy. Curr Opin HIV AIDS. 2016 Jul;11(4):417-423.

43. Fromentin $R$, Bakeman $W$, Lawani MB, et al. CD4+ T cells expressing PD-1, TIGIT and LAG-3 contribute to HIV persistence during ART. PLoS Pathog. 2016 Jul 14;12(7):e1005761.

44. Procopio FA, Fromentin R, Kulpa DA, et al. A novel assay to measure the magnitude of the inducible viral reservoir in HIV-infected individuals. EBioMedicine. 2015 Jun 27;2(8):874-883.

45. Vandergeeten C, Fromentin R, Merlini E, et al. Cross-clade ultrasensitive PCR-based assays to measure HIV persistence in largecohort studies. J Virol. 2014 Nov;88(21):12385-12396.

46. Lorenzo-Redondo R, Fryer HR, Bedford T, et al. Persistent HIV-1 replication maintains the tissue reservoir during therapy. Nature. 2016 Feb 4;530(7588):51-56.

47. Huang $Y$, Hoque MT, Jenabian M-A, et al. Antiretroviral drug transporters and metabolic enzymes in human testicular tissue: potential contribution to HIV-1 sanctuary site. J Antimicrob Chemother. 2016 Jul;71(7):1954-1965.

48. Madruga JR, Cassetti I, Suleiman JMAH, et al. The safety and efficacy of switching stavudine to tenofovir $\mathrm{df}$ in combination with lamivudine and efavirenz in HIV-1-infected patients: threeyear follow-up after switching therapy. HIV Clin Trials. 2007;8 (6):381-390.

49. Ucciferri C, Falasca K, Vignale F, et al. Improved metabolic profile after switch to darunavir/ritonavir in HIV positive patients previously on protease inhibitor therapy. J Med Virol. 2013;85(5):755759.

50. Mondi A, Fabbiani M, Ciccarelli N, et al. Efficacy and safety of treatment simplification to atazanavir/ritonavir + lamivudine in HIV-infected patients with virological suppression: 144 week follow-up of the AtLaS pilot study. J Antimicrob Chemother. 2015;70 (6):1843-1849.

51. Rockstroh JK, DeJesus E, Lennox JL, et al. Durable efficacy and safety of raltegravir versus efavirenz when combined with tenofovir/emtricitabine in treatment-naive HIV-1-infected patients: final 5year results from STARTMRK. J Acquir Immune Defic Syndr. 2013;63 (1):77-85.

52. Maclnnes A, Lazzarin A, Di Perri G, et al. Maraviroc can improve lipid profiles in dyslipidemic patients with HIV: results from the MERIT trial. HIV Clin Trials. 2011;12(1):24-36.

53. Horton JD, Goldstein JL, Brown MS. SREBPs: activators of the complete program of cholesterol and fatty acid synthesis in the liver. J Clin Invest. 2002 May;109(9):1125-1131.

54. Bianchi S, Grimaldi D, Bigazzi R. Statins and lipid-lowering strategies in cardiorenal patients. Contrib Nephrol. 2011;171:143-150.

55. Blanco-Colio LM, Tuñón J, Martín-Ventura JL, et al. Antiinflammatory and immunomodulatory effects of statins. Kidney Int. 2003;63(1):12-23.

56. Elahi $\mathrm{S}$, Weiss RH, Merani S. Atorvastatin restricts HIV replication in CD4+ T cells by upregulation of p21. AIDS. 2016 Jan;30(2):171-183.

57. Calza L, Vanino E, Salvadori C, et al. Tenofovir/emtricitabine/efavirenz plus rosuvastatin decrease serum levels of inflammatory markers more than antiretroviral drugs alone in antiretroviral therapynaive HIV infected patients. HIV Clin Trials. 2014;15:1-13.

58. Aslangul E, Fellahi S, Assoumou LK, et al. High-sensitivity C-reactive protein levels fall during statin therapy in HIV-infected patients receiving ritonavir-boosted protease inhibitors. AIDS. 2011;25:1128-1131.

59. Nakanjako D, Ssinabulya I, Nabatanzi R, et al. Atorvastatin reduces T-cell activation and exhaustion among HIV-infected CART-treated suboptimal immune responders in Uganda: a randomised crossover placebo-controlled trial. Trop Med Int Health. 2015 Mar;20 (3):380-390.

60. Ganesan A, Crum-Cianflone N, Higgins J, et al. High dose atorvastatin decreases cellular markers of immune activation without affecting HIV-1 RNA levels: results of a double-blind randomized placebo controlled clinical trial. J Infect Dis. 2011 Mar 15;203 (6):756-764
61. Hileman CO, Turner R, Funderburg NT, et al. Changes in oxidized lipids drive the improvement in monocyte activation and vascular disease after statin therapy in HIV. AIDS. 2016 Jan 2;30(1):65-73.

62. Nou E, Lu MT, Looby SE, et al. Serum oxidized low-density lipoprotein decreases in response to statin therapy and relates independently to reductions in coronary plaque in patients with HIV. AIDS. 2016 Feb 20;30(4):583-590.

63. Funderburg NT, Jiang Y, Debanne SM, et al. Rosuvastatin reduces vascular inflammation and T-cell and monocyte activation in HIV infected subjects on antiretroviral therapy. J Acquir Immune Defic Syndr. 2015 Apr 1; 68(4):396-404..

-. The preliminary results of this study stimulate to use rosuvastatin as an active agent in the complex contest of immune activation, not only for dyslipidemia, but also in a more inclusive immunological sight

64. Erlandson KM, O'Riordan M, Labbato D, et al. Relationships between inflammation, immune activation, and bone health among HIV-infected adults on stable antiretroviral therapy. J Acquir Immune Defic Syndr. 2014;65:290-298.

65. Funderburg NT, Boucher M, Sattar A, et al. Rosuvastatin decreases intestinal fatty acid binding protein (I-FABP), but does not alter zonulin or lipopolysaccharide binding protein (LBP) levels, in HIVinfected subjects on antiretroviral therapy. Pathog Immun. 2016 Spring;1(1):118-128.

66. Antithrombotic Trialists' (ATT) Collaboration, Baigent C, Blackwell L, Collins $\mathrm{R}$, et al. Aspirin in the primary and secondary prevention of vascular disease: collaborative meta-analysis of individual participant data from randomised trials. Lancet. 2009;373(9678):18491860.

67. O'Brien M, Montenont $\mathrm{E}, \mathrm{Hu} \mathrm{L}$, et al. Aspirin attenuates platelet activation and immune activation in HIV-1-infected subjects on antiretroviral therapy: a pilot study. J Acquir Immune Defic Syndr. 2013;63(3):280-288.

- This study shows a decrease in terms of platelet activation after just 1 week of aspirin in HIV patient, that have an increased cardiovascular risk independently from the presence of other risk factors.

68. Liu SF, Wang H, Li Z-J, et al. Aspirin induces lytic cytotoxicity in Epstein-Barr virus-positive cells. Eur J Pharmacol. 2008;589(13):8-13.

69. Keller SA, Schattner EJ, Cesarman E. Inhibition of NF-kappaB induces apoptosis of KSHV-infected primary effusion lymphoma cells. Blood. 2000;96(7):2537-2542.

70. Lei $X$, Bai $Z$, Ye F, et al. Regulation of NF-kappaB inhibitor IkappaBalpha and viral replication by a KSHV microRNA. Nat Cell Biol. 2010;12(2):193-199.

71. Ziegler HK, Unanue ER. Decrease in macrophage antigen catabolism caused by ammonia and chloroquine is associated with inhibition of antigen presentation to T cells. Proc Natl Acad Sci USA. 1982;79(1):175-178.

72. Sperber K, Quraishi H, Kalb TH, et al. Selective regulation of cytokine secretion by hydroxychloroquine: inhibition of interleukin 1 alpha (IL-1-alpha) and IL-6 in human monocytes and T cells. J Rheumatol. 1993;20(5):803-808.

73. Loffler BM, Bohn E, Hesse B, et al. Effects of antimalarial drugs on phospholipase A and lysophospholipase activities in plasma membrane, mitochondrial, microsomal and cytosolic subcellular fractions of rat liver. Biochim Biophys Acta. 1985;835(3):448-455.

74. Cohen SN, Yielding KL. Spectrophotometric studies of the interaction of chloroquine with deoxyribonucleic acid. J Biol Chem. 1965:240:3123-3131.

75. Lesiak A, Narbutt J, Sysa-Jedrzejowska A, et al. Effect of chloroquine phosphate treatment on serum MMP-9 and TIMP-1 levels in patients with systemic lupus erythematosus. Lupus. 2010;19 (6):683-688.

76. Kyburz D, Brentano F, Gay S. Mode of action of hydroxychloroquine in RA - evidence of an inhibitory effect on toll-like receptor signaling. Nat Clin Pract Rheumatol. 2006;2(9):458-459. 
77. Fesen MR, Kohn KW, Leteurtre F, et al. Inhibitors of human immunodeficiency virus integrase. Proc Natl Acad Sci USA. 1993;90:23992403.

78. Jiang MC, Lin JK, Chen SS. Inhibition of HIV-1 Tat transactivation by quinacrine and chloroquine. Biochem Biophys Res Commun. 1996;226:1-7.

79. Piconi S, Parisotto S, Rizzardini G, et al. Hydroxychloroquine drastically reduces immune activation in HIV-infected, antiretroviral therapy-treated immunologic nonresponders. Blood. 2011;118 (12):3263-3272.

80. Routy J-P, Angel JB, Patel M, et al. Assessment of chloroquine as a modulator of immune activation to improve CD4 recovery in immune nonresponding HIV-infected patients receiving antiretroviral therapy. HIV Med. 2015;16(1):48-56.

81. He J, Chen Y, Farzan M, et al. CCR3 and CCR5 are co-receptors for HIV-1 infection of microglia. Nature. 1997;385:645-649.

82. Kusao I, Shiramizu B, Liang C-Y, et al. Cognitive performance related to HIV-1-infected monocytes. J Neuropsychiatry Clin Neurosci. 2012;24:71-80.

83. Westhorpe CLV, Maisa A, Spelman T, et al. Associations between surface markers on blood monocytes and carotid atherosclerosis in HIV-positive individuals. Immunol Cell Biol. 2014;92:133-138.

84. Funderburg NT, Zidar DA, Shive $C$, et al. Shared monocyte subset phenotypes in HIV-1 infection and in uninfected subjects with acute coronary syndrome. Blood. 2012;120:4599-4608.

- The finding of a relation between monocyte phenotypes in HIV patients and in cardiovascular patient suggests the necessity to prevent cardiovascular events independently from other vascular risk factors.

85. Baker JV, Hullsiek KH, Singh A, et al. Immunologic predictors of coronary artery calcium progression in a contemporary HIV cohort. AIDS. 2013;28:831-840.

86. Brown KC, Patterson KB, Malone SA, et al. Single and multiple dose pharmacokinetics of maraviroc in saliva, semen, and rectal tissue of healthy HIV-negative men. J Infect Dis. 2011;203:1484-1490.

87. Scopelliti F, Pollicita M, Ceccherini-Silberstein F, et al. Comparative antiviral activity of integrase inhibitors in human monocyte-derived macrophages and lymphocytes. Antiviral Res. 2011;92:255-261.

88. Marsden MD, Avancena $P$, Kitchen $C M$, et al. Single mutations in HIV integrase confer high-level resistance to raltegravir in primary human macrophages. Antimicrob Agents Chemother. 2011;55:3696-3702.

89. Jiang $W$, Lederman MM, Hunt $P$, et al. Plasma levels of bacterial DNA correlate with immune activation and the magnitude of immune restoration in persons with antiretroviral-treated HIV infection. J Infect Dis. 2009;199(8):1177-1185.

90. Funderburg NT, Mayne E, Sieg SF, et al. Increased tissue factor expression on circulating monocytes in chronic HIV infection: relationship to in vivo coagulation and immune activation. Blood. 2010;115(2):161-167.

91. Zevin AS, McKinnon L, Burgener A, et al. Microbial translocation and microbiome dysbiosis in HIV-associated immune activation. Curr Opin HIV AIDS. 2015;11:182-190.

- Not only microbial translocation is responsible for immune activation, but also the modified gut flora that characterize HIV patients, giving a more complex point of view of the problem and stimulating more studies for other solutions.

92. Dillon SM, Lee EJ1, Kotter CV, et al. An altered intestinal mucosal microbiome in HIV-1 infection is associated with mucosal and systemic immune activation and endotoxemia. Mucosal Immunol. 2014;7:983-994.

93. Vujkovic-Cvijin I, Dunham RM, Iwai S, et al. Dysbiosis of the gut microbiota is associated with HIV disease progression and tryptophan catabolism. Sci Transl Med. 2013;5:193ra191.

94. Mutlu EA, Keshavarzian A, Losurdo J, et al. A compositional look at the human gastrointestinal microbiome and immune activation parameters in HIV infected subjects. PLoS Pathog. 2014;10: e1003829.

95. Gori A, Tincati C, Rizzardini G, et al. Early impairment of gut function and gut flora supporting a role for alteration of gastrointestinal mucosa in human immunodeficiency virus pathogenesis. J Clin Microbiol. 2008;46:757-758.

96. Dagenais-Lussier X, Aounallah M, Mehraj V, et al. Kynurenine reduces memory CD4 T-cell survival by interfering with interleukin-2 signaling early during HIV-1 infection. J Virol. 2016 Aug 12;90 (17):7967-7979.

97. Lee SA, Mefford JA, Huang Y, et al. Host genetic predictors of the kynurenine pathway of tryptophan catabolism among treated HIVinfected Ugandans. AIDS. 2016 Jul 17;30(11):1807-1815.

98. Boasso A, Vaccari M, Hryniewicz A, et al. Regulatory T-cell markers, indoleamine 2,3-dioxygenase, and virus levels in spleen and gut during progressive simian immunodeficiency virus infection. J Virol. 2007 Nov 12;81(21):11593-11603.

99. Routy J-P, Mehraj V, Vyboh K, et al. Clinical relevance of kynurenine pathway in HIV/AIDS: an immune checkpoint at the crossroads of metabolism and inflammation. AIDS Rev. 2015 Apr-Jun;17(2):96106.

100. Jenabian M-A, El-Far M, Vyboh K, et al. Immunosuppressive tryptophan catabolism and gut mucosal dysfunction following early HIV infection. J Infect Dis. 2015 Aug 1;212(3):355-366.

101. Vujkovic-Cvijin I, Dunham RM, Iwai S, et al. Dysbiosis of the gut microbiota is associated with HIV disease progression and tryptophan catabolism. Sci Transl Med. 2013 Jul 10;5(193):193ra91.

102. Roberfroid M. Prebiotics: the concept revisited. J Nutr. 2007;137(3 Suppl 2):830S-7S.

103. Guggenbichler JP, De Bettignies-Dutz A, Meissner P. Acidic oligosaccharides from natural sources block adherence of Escherichia coli on uroepithelial cells. Pharm Pharmacol Lett. 1997;7:35-38.

104. Hummelen R, Vos AP, Van't Land B, et al. Altered host-microbe interaction in HIV: a target for intervention with pro- and prebiotics. Int Rev Immunol. 2010 Oct;29(5):485-513.

105. Kelly P, Todd J, Sianongo S, et al. Susceptibility to intestinal infection and diarrhoea in Zambian adults in relation to HIV status and CD4 count. BMC Gastroenterol. 2009;9:7.

106. Plettenberg A, Stoehr A, Stellbrink H-J, et al. A preparation from bovine colostrum in the treatment of HIV-positive patients with chronic diarrhea. Clin Investig. 1993;71(1):42-45.

107. Gori A, Rizzardini G, Van't Land B, et al. Specific prebiotics modulate gut microbiota and immune activation in HAART-naive HIVinfected adults: results of the "COPA" pilot randomized trial. Mucosal Immunol. 2011;4(5):554-563.

- Modulating gut microbiota and consequently immune activation could be an interesting way to reduce immune activation without adding other drugs in the already chronically medicalized HIV population.

108. Cahn P, Ruxrungtham K, Gazzard B, et al. The immunomodulatory nutritional intervention NR100157 reduced CD4(+) T-cell decline and immune activation: a 1-year multicenter randomized controlled double-blind trial in HIV-Infected persons not receiving antiretroviral therapy (the BITE study). CID. 2013;57:139-146.

109. Byakwaga H, Kelly M, Purcell DFJ, et al. Intensification of antiretroviral therapy with raltegravir or addition of hyperimmune bovine colostrum in HIV-infected patients with suboptimal CD4+ T-cell response: a randomized controlled trial. J Infect Dis. 2011;204 (10):1532-1540.

110. Food and Agriculture Organization of the United Nations (FAO). Health and nutritional properties of probiotics in food including powder milk with live lactic acid bacteria. 2001. Available from: www.who.int/foodsafety/publications/fs_management/en/probio tics.pdf

111. Kaila M, Isolauri E, Soppi E, et al. Enhancement of the circulating antibody secreting cell response in human diarrhea by a human Lactobacillus strain. Pediatr Res. 1992;32:141-144.

112. Schlee M, Wehkamp J, Altenhoefer A. Induction of human betadefensin 2 by the probiotic Escherichia coli Nissle 1917 is mediated through flagellin. Infect Immun. 2007;75:2399-2407.

113. Spinler JK, Taweechotipatr M, Rognerud CL, et al. Human-derived probiotic Lactobacillus reuteri demonstrate antimicrobial activities targeting diverse enteric bacterial pathogens. Anaerobe. 2008;14:166-171. 
114. Heinemann C, Van Hylckama Vlieg JE, Janssen DB, et al. Purification and characterization of a surface-binding protein from Lactobacillus fermentum RC-14 that inhibits adhesion of Enterococcus faecalis 1131. FEMS Microbiol Lett. 2000;190:177-180.

115. Alak Jl, Wolf BW, Mdurvwa EG. Effect of Lactobacillus reuteri on intestinal resistance to Cryptosporidium parvum infection in a murine model of acquired immunodeficiency syndrome. J Infect Dis. 1997;175:218-221.

116. Su Y, Zang B, Su L. CD4 detected from Lactobacillus helps understand the interaction between Lactobacillus and HIV. Microbiol Res. 2013 Jun 12;168(5):273-277.

117. González-Hernández LA, Jave-Suarez L-S, Fafutis-Morris $M$, et al. Synbiotic therapy decreases microbial translocation and inflammation and improves immunological status in HIV-infected patients: a doubleblind randomized controlled pilot trial. Nutr J. 2012 Oct;11(1):90.

118. Hemsworth JC, Hekmat SHekmid GReid S, Reid G. Micronutrient supplemented probiotic yogurt for HIV-infected adults taking HAART in London, Canada. Gut Microbes. 2012;3(5):414-419.

119. d'Ettorre G, Ceccarelli G, Giustini N, et al. Probiotics reduce inflammation in antiretroviral treated, HIV-infected individuals: results of the "Probio-HIV" clinical trial. PLoS One. 2015;10(9): e0137200.

120. Kim CJ, Walmsley SL, Raboud JM, et al. Can probiotics reduce inflammation and enhance gut immune health in people living with HIV: study designs for the probiotic Visbiome for inflammation and translocation (PROOV IT) pilot trials. HIV Clin Trials. 2016 Jul; 17 (4):147-157.

121. Hensley-McBain T1, Zevin AS, Manuzak J, et al. Effects of fecal microbial transplantation on microbiome and immunity in simian immunodeficiency virus-infected macaques. J Virol. 2016 Apr 29;90 (10):4981-4989.

122. Navarro-Gonzalez JF, Mora-Fernandez C, Muros de Fuentes M, et al. Effect of phosphate binders on serum inflammatory profile, soluble CD14, and endotoxin levels in hemodialysis patients. Clin J Am Soc Nephrol. 2011;6:2272-2279.

123. Ferramosca E, Burke S, Chasan-Taber S, et al. Potential antiatherogenic and anti-inflammatory properties of sevelamer in maintenance hemodialysis patients. Am Heart J. 2005;149:820-825.

- This study shows pleiotropic activity (atherogenesis, antiinflammatory effect, and reduction of endotoxin levels) of a nephrologic drug, that deserve further insights in HIV population, because of the presence of little and conflicting results.

124. Sandler NG, Zhang X, Bosch RJ, et al. Sevelamer does not decrease lipopolysaccharide or soluble CD14 levels but decreases soluble tissue factor, low-density lipoprotein (LDL) cholesterol, and oxidized LDL cholesterol levels in individuals with untreated HIV infection. J Infect Dis. 2014 Nov 15;210(10):1549-1554.

125. Kristoff J,Haret-Richter G, et al. Early microbial translocation blockade reduces SIV-mediated inflammation and viral replication. J Clin Invest. 2014 Jun; 124 (6): 2802-2806 